\title{
Norepinephrine elicitation of aggressive display responses in Betta splendens
}

ROBERT L. MARRONE, SIDNEY L. PRAY AND CECIL C. BRIDGES

TEXAS CHRISTIAN UNIVERSITY

From arguments elucidated by D. H. Funkenstein and others, it was hypothesized that the aggressive display of the Siamese fighting fish was under hormonal (i.e., norepinephrine) control. Eighteen visually isolated fish were observed for $10 \mathrm{~min}$. each under a plain water control condition and with norepinephrine bitartrate solutions in their individual living tanks. Significant increases in the frequency of fin extensions and gill flaring responses, as well as increased coloration were found for the drug group. Six fish treated with epinephrine bitartrate resembled fish under the control condition except for color change; all six bleached during observation.

Numerous comparisons of the pharmacological actions of norepinephrine and epinephrine, both in vivo and in vitro, have been reported. In general, these studies have shown norepinephrine to be the major adrenergic mediator at postganglionic neuroeffector junctions and epinephrine to be the predominant sympathomimetic amine released by the adrenal medulla (Goodman \& Gilman, 1960)。Funkenstein (1955) and others have suggested that aggressive behavior is correlated with norepinephrine release in the species and in individuals within species and that epinephrine release is associated with flight responses or nonaggressive responses. In addition, epinephrine has been related to a wide variety of emotional responses to stressful situations. Pray (1965) has recently shown that intraperitonially injected norepinephrine increased reflexive fighting in response to aversive stimulation in rats. The same animals treated with epinephrine did not manifest an increase in the frequency of aggressive responses.

Male Siamese fighting fish, Betta splendens, emit a highly stereotyped aggressive display pattern prior to attack when presented with another male of the species or with their mirror image. This pattern typically consists of an air gulp, approach to the other fish or image, a deepening of coloration, fin extension, and a definitive flaring of the gills. The components of this display, although typically occurring together, may occur independently or in any other combination. Based on an exploratory study by Marrone, in which large amounts of norepinephrine bitartrate were found to elicit in vacuo gill flaring in several fish, it was hypothesized that all the components of the agonistic display (air gulps, gill flaring, fin extension, and color change) would increase in frequency in fish treated with norepinephrine compared with their responses under a non-treated control condition. It was also hypothesized that the responses of the fish treated with epinephrine would be qualitatively different from the responses of untreated fish or fish treated with norepinephrine.

\section{Method}

Fish were observed in 2 qt. glass living tanks. These tanks were shelved in rows visually isolated from each other by a paraffin coating on the ends of the tanks. Visual isolation from the laboratory environment was provided by sheets of translucent plastic surrounding the shelves. Fish were observed through peep holes in the translucent screen, which were $1 \mathrm{ft}$, from the tanks. Light was provided by 40 watt fluorescent lights $1 \mathrm{ft}$. above the tanks. These lights were turned on about 10 min, before each observation session began. The laboratory was darkened to eliminate observer shadows. Before the observation period, the water in the tanks of those fish to be observed was changed to $500 \mathrm{ml}$ of fresh water.

Eighteen mature male fighting fish were observed for ten minutes each in a norepinphrine bitartrate solution. A similar control observation of each fish was made in plain water. Half the fish were observed first under the control condition and at least one week later in the norepinephrine bitartrate solution, for the other half, the control and drug observation periods were reversed. Three drug levels, 35, 70, and $140 \mathrm{mg}$ of norepinephrine bitartrate in $500 \mathrm{ml}$ of water were used, with three fish in each of the previously mentioned half-groups receiving one of the levels of the drug. Two (sometimes three) observers simultaneously watched a single fish for each observation period. Consensus was obtained on all notes recorded. All air gulps, gill flare responses, and changes in coloration were noted along with observations of swimming activity, fin position, and changes in the general appearance of the fish. Six additional fish, with similar amounts of epinephrine bitartrate in their water were observed under this condition.

\section{Results and Discussion}

From inspection of the data, it was obvious that tests of significance of either drug level or drug-control order effects would be gratuitous; they were obviously non-significant. However, the overall difference between the norepinephrine and water-control treatments was striking; none of the fish in the water-control condition was observed to flare, whereas 9 of the 18 fish spontaneously flared with norepinephrine treatment. The difference between the two conditions is significant beyond the .005 level of confidence. Of the 16 non-zero differences between color changes (hue change or increase in saturation) in fish in the control and norepine- 
phrine treatment conditions, 14 favor the norepinephrine condition $(p<.005)$. Of 11 non-zero differences in fin extension change, 9 favor the norepinephrine group $(p<.05)$. The difference in air gulps under norepinephrine and control conditions was not significant, as was the difference in activity level under the two conditions.

No incidence of gill flaring or fin extension was recorded for fish in the epinephrine bitartrate group. In addition, there was no significant difference in frequency of air gulps between the epinephrine and control groups. In general, the six fish treated with epinephrine bitartrate resembled fish in the control condition except for color change; all six bleached $(p<.05)$.

The gill flaring response is rarely encountered in the visually isolated fish and the authors have found no reference to its spontaneous elicitation with non-visual stimuli. It would appear that this component of the agonistic display, as well as fin extension and increased coloration, are also specifically elicited by norepinephrine since the highly similar epinephrine does not produce them. These component responses are also not a function of an increased activation level which would make the fish generally more responsive to all stimuli; general activity levels in the norepinephrine and control conditions were similar.

\section{References}

Goodman, L. S.. \& Gilman. A. Pharmacolorical basis of therapeutics, New York: Macmillan Co., 1960.

Funkenstein, E. H. The physiology of fear and anger. Scicnt. American, 1955, 192, 74-80.

Pray, S. L. Effects of adrenal in and noradrenalin on reflexive fighting in response to aversive stimulation in the white rat 1965, (in preparation) 